Mr Nikola Bračkta, potpukovnik, dipl. inž.

\section{PROGRAMSKI SISTEM ZA ODREĐIVANJE PROIZVODNE RADNE SNAGE REMONTNIH JEDINICA I USTANOVA VJ \\ - II deo -}

\begin{abstract}
Clanak predstavlja nastavak članka objavljenog u prethodnom broju VTG pod istim naslovom. Na hipotetickom primeru šeme oslanjanja, materijalne formacije i normativa vremena prikazuje se način korisćenja programskog sistema kao modela remontne jedinice za eksperimentisanje, radi iznalaženja adekvatnog lǐ̌nog sastava proizvodne radne snage.
\end{abstract}

\section{Uvod}

Programski sistem, koji je pod istim naslovom prikazan $\mathrm{u}$ prethodnom broju VTG-a, realizovan je u COBOL-u još 1985. na računaru $\mathrm{H}-6$, a po zahtevu TU.

Imajući $u$ vidu potrebe za primenu ovog programskog sistema i sadašnju opremljenost personalnim računarima, autor ga je realizovao $u$ programskom jeziku dBASE IV. Time je programski sistem postao dostupan širem krugu korisnika i, pored osnovne, može imati i edukativnu namenu $U$ osnovi, sistem omogućuje proračun broja proizvodnih radnika različitih kategorija lica uvek kad je potrebno preispitati postojeću ličnu formaciju zbog novonastalih uslova.

$\mathrm{Na}$ jednom hipotetickom primeru bice prikazan način formiranja baze ulaznih podataka, proračun, rezultati proračuna, kao i primer izvođenja eksperimenata, kada se računarski sistem tretira kao model remontne jedinice. Eksperimentisanje će se sprovesti za slučajeve promene prosčnog sedmičnog radnog vremena $(42,40$ i 35 časova). $\mathrm{Na}$ bazi sprovedenih eksperimenata biće doneta ocena adekvatnosti postojeće lične formacije tretirane remontne jedinice.

\section{Formiranje ulazne baze podataka}

Jedinica ili ustanova tehničkog održavanja (u daljem tekstu remontna jedinica) vrši laki ili srednji remont $u$ miru i ratu, što se, u smislu ovog članka, naziva vid i nvo remonta. Vid i nivo remonta kodirani su kao što je prikazano u prvoj koloni tabele 7, a baza podataka se formira za remontne jedinice čije su Šifre i nazivi dati u tabeli 1 .

$\mathrm{Na}$ svaku remontnu jedinicu lakog remonta u miru ili ratu može biti oslonjena jedna ili više jedinica različitog nivoa organizacije (jedinice sa samostalnom formacijom $i$ niži organizacijski nivoi). Hipotetički primer šeme oslanjanja po remontu prikazan je na slici 1, a na osnovu nje formirana je tabela 2.

Sifre u kolonama SIF1, SIF2 i SIF3 u tabeli predstavljaju šifre remontnih jedinica vida i nivoa koji je šifrovano dat $\mathrm{u}$ kolonama V1, V2 i V3 respektivno (prema tabeli 7). SIF4 predstavlja šifru oslonjene jedinice, a KLAS njemu klasifikaciju (A, B, R i G). Klasifikacija oslonjenih jedinica $u$ ratu uzima se kao G (FMS su 100\% u eksploataciji). Iako klasifikacija B ne egzistira, ona omogućava da se preko nje izraze neki drugi pretpostavljeni odnosi FMS u eksploataciji i u ratnoj rezervi. U koloni V1 moguće su vrednosti 2 ili 5, u koloni V2 vrednosti 1 i 4, a kolona 


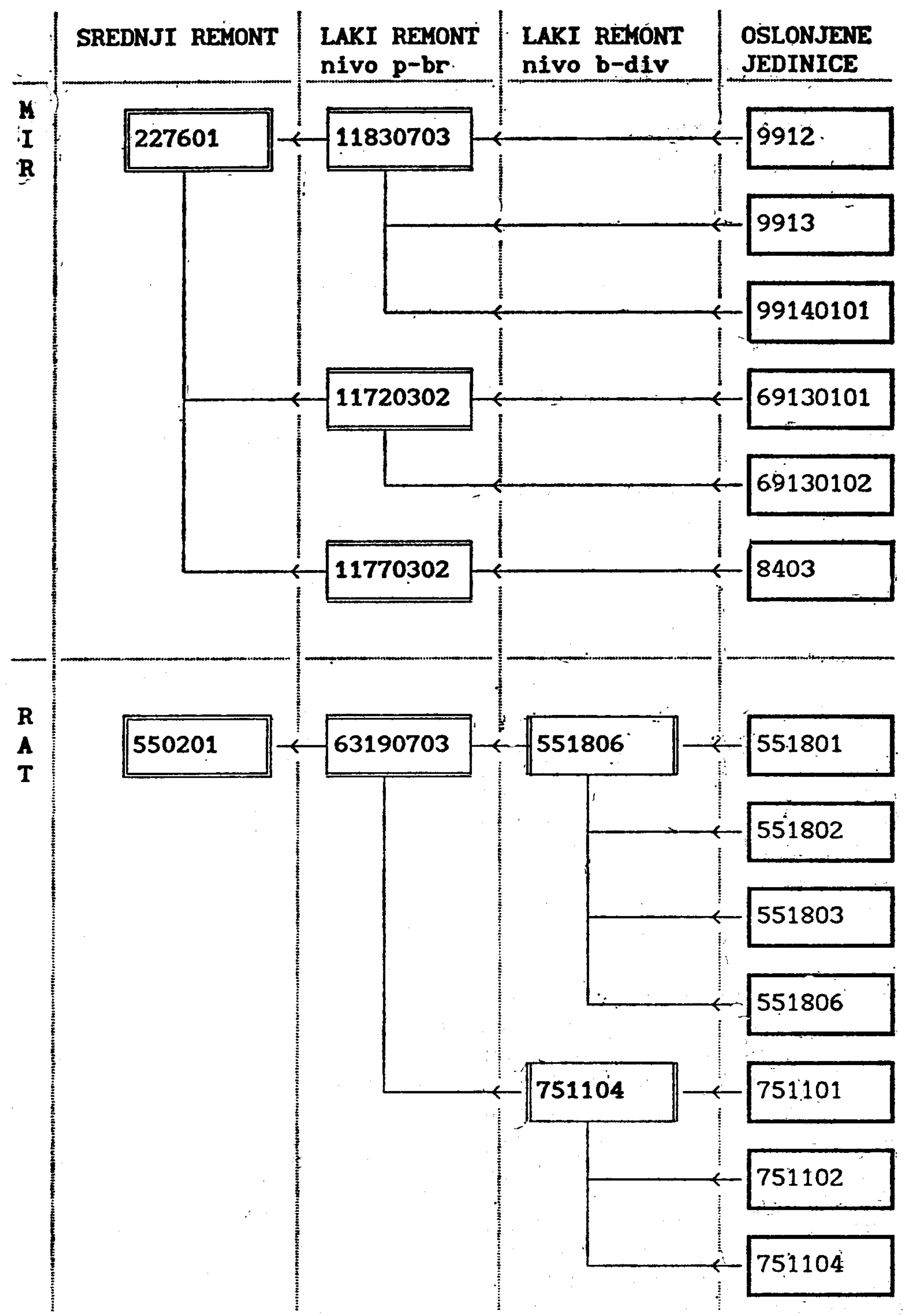

Sl. 1 - Sema oslanjanja jedinica po remontu 
Sifarnik jedinica

\begin{tabular}{|l|l|}
\hline SIF_JED & NAZ_JED \\
\hline \hline 227601 & $43 \cdot$ CSR \\
11830703 & $92 \cdot$ VTO \\
11720302 & $13 \cdot$ VTO \\
550201 & $72 \cdot$.SR \\
63190703 & $52 \cdot$ VTO \\
551806 & $14 \cdot$ OdTOd \\
751104 & $35 \cdot$ OdTOd \\
9912 & $129 \cdot \mathrm{mbr}$ \\
9913 & $34 \cdot \mathrm{mbr}$ \\
99140101 & $1-1 \cdot \mathrm{mb} / 47 \cdot \mathrm{mbr}$ \\
69130101 & $1-1 \cdot \mathrm{pb} / 96 \cdot \mathrm{mtbr}$ \\
69130102 & $2-1 \cdot \mathrm{pb} / 96 \cdot \mathrm{mtbr}$ \\
8403 & $194 \cdot \mathrm{mabr}$ \\
551801 & $1.0 \mathrm{~kb} / 74 \cdot$ okbr \\
551802 & $2 \cdot$ okb/74.Okbr \\
551803 & mb/74.0kbr \\
751101 & $1 \cdot$ inzb/13.inzp \\
751102 & $2 \cdot$ inzb/13.inzp \\
\hline
\end{tabular}

Podaci o oslanjanju

Tabela 2

\begin{tabular}{|l|l|l|l|l|l|l|l|}
\hline SIF1 & V1 & SIF2 & V2 & SIF3 & V3 & SIF4 & KLAS \\
\hline \hline 227601 & 2 & 11830703 & 1 & & 0 & 9912 & A \\
227601 & 2 & 11830703 & 1 & & 0 & 9913 & A \\
227601 & 2 & 11830703 & 1 & & 0 & 99140101 & A \\
227601 & 2 & 11720302 & 1 & & 0 & 69130101 & A \\
227601 & 2 & 11720302 & 1 & & 0 & 69130102 & R \\
227601 & 2 & 11770302 & 1 & & 0 & 8403 & R \\
550201 & 5 & 63190703 & 4 & 551806 & 3 & 551801 & G \\
550201 & 5 & 63190703 & 4 & 551806 & 3 & 551802 & G \\
550201 & 5 & 63190703 & 4 & 551806 & 3 & 551803 & G \\
550201 & 5 & 63190703 & 4 & 551806 & 3 & 551806 & G \\
550201 & 5 & 63190703 & 4 & 751104 & 3 & 751101 & G \\
550201 & 5 & 63190703 & 4 & 751104 & 3 & 751102 & G \\
550201 & 5 & 63190703 & 4 & 751104 & 3 & 751104 & G \\
\hline
\end{tabular}

V3 ostavlja se blanko (za mirnodopske remontne jedinice) ili se popunjava vrednošću 3. Sifra jedinice (remontne il oslonjene) formira se od matičnog broja jedinice sa samostalnom formacijom (četiri pozicije) i rednih brojeva bataljonskog $i$ četnog satava $u$ organizacijskoj strukturi (po dve pozicije).

Svaka oslonjena jedinica ima svoju materijalnu formaciju koja je za posmatrani primer prikazana u tabeli 3 .

Realno je pretpostaviti da i remontne jedinice imaju svoja FMS, te se one mogu osloniti same na sebe ili na neke druge remontne jedinice, zavisno od specifičnosti FMS. Tako je jedinica lakog remonta u miru sa šifrom $\gg 751104 \ll$ oslonjena na sebe (SIF3=SIF4, tab. 3).

Zavisno od klasifikacije oslonjene jedinice, njena FMS su u većem ili manjem procentu $u$ eksploataciji, a ostatak se čuva u ratnoj rezervi ili se jedinica delom pounjava iz popisanog fonda. $\mathrm{Za}$ neke klasne FMS, kojima se jedinice delom popunjavaju iz popisanog fonda, odnosi FMS u eksploataciji i u ratnoj rezervi su različiti za pojedine klase jedinica. Pretpostavljeni koeficijenti koji izražavaju te odnose prikazani su $u$ tabeli 4 . 
Podaci o materijalnoj formaciji jedinica

\begin{tabular}{|l|l|r|}
\hline SIF & FMS & KOI \\
\hline 9912 & 11101 & 214 \\
9912 & 11102 & 234 \\
9912 & 12101 & 45 \\
9912 & 12202 & 38 \\
9913 & 11101 & 235 \\
9913 & 11102 & 193 \\
9913 & 12101 & 81 \\
9913 & 12202 & 73 \\
99140101 & 11101 & 234 \\
99140101 & 11102 & 124 \\
99140101 & 12101 & 5 \\
99140101 & 12302 & 3 \\
69130101 & 11101 & 456 \\
69130101 & 11102 & 742 \\
69130101 & 12101 & 35 \\
69130101 & 12302 & 19 \\
69130102 & 11101 & 923 \\
69130102 & 11102 & 234 \\
69130102 & 12101 & 56 \\
69130102 & 12202 & 47 \\
8403 & 11101 & 234 \\
8403 & 11101 & 346 \\
8403 & 12101 & 231 \\
8403 & 12202 & 384 \\
551801 & 12301 & 234 \\
551801 & 12302 & 354 \\
551802 & 12101 & 324 \\
551802 & 12202 & 210 \\
551803 & 11102 & 825 \\
551803 & 12301 & 286 \\
551806 & 11101 & 35 \\
551806 & 11102 & 24 \\
551806 & 12301 & 4 \\
751101 & 11101 & 843 \\
751101 & 11102 & 546 \\
751102 & 12201 & 89 \\
751102 & 12302 & 94 \\
751104 & 11101 & 923 \\
751104 & 11102 & 234 \\
751104 & 12202 & 35 \\
\hline
\end{tabular}

Svako FMS iz tabele 3 zahteva jednu li više vojnoevidencionih specijalnosti (VES) tehničke službe za njegovo tehničko održavanje. One mogu da budu različitih kategorija kadra (vojnici, podoficiri, oficiri ili civilna lica u VJ) i razl\}čite kvalifikacije (uslovno $\mathrm{KV}$ i VKV). Primer je pojednostavljen tako da se vojnici tretiraju kao KV, podoficiri i oficiri kao VKV, a civilna lica kao KV i VKV izvršioci remonta.
Koeficijenti odnosa FMS u eksploataciji $i$ ratnoj rezervi za pojedine klasifikacije jedinica

\begin{tabular}{|l|l|r|r|}
\hline EMS & KLAS & KE & KRR \\
\hline \hline 121 & A & 0.50 & 0.20 \\
122 & A & 0.20 & 0.10 \\
123 & A & 0.50 & 0.20 \\
999 & A & 0.70 & 0.30 \\
121 & B & 0.20 & 0.50 \\
122 & B & 0.10 & 0.50 \\
123 & B & 0.20 & 0.50 \\
999 & B & 0.30 & 0.70 \\
121 & R & 0.07 & 0.63 \\
122 & R & 0.05 & 0.25 \\
123 & R & 0.07 & 0.63 \\
999 & R & 0.10 & 0.90 \\
121 & G & 1.00 & 0.00 \\
122 & G & 1.00 & 0.00 \\
123 & G & 1.00 & 0.00 \\
999 & G & 1.00 & 0.00 \\
\hline
\end{tabular}

Prosečno godišnje vreme angažovanja je različito za pojedine kategorije kadra, vidove $i$ nivoe remonta i prikazano je u tabeli 5. Podaci iz kolone VREME odnose se na 42-časovno nedeljno radno vreme, a iz kolona T40 i T35 na 40-časovno i 35-časovno nedeljno radno vreme. Program operiše samo podacima iz kolone VREME, a sve tri zajedno predstavljaju eksperimentalni plan, tj. varijante zo koje ce se vršiti proračun.

Tabela 5

Prosečno godišnje vreme angažovanja kategorija kadra za pojedine nivoe $i$ vidove reinurata.

\begin{tabular}{|l|l|r|r|r|}
\hline NIV & KAT & VREME & T40 & T35 \\
\hline \hline 1 & 1 & 800 & 762 & 667 \\
1 & 2 & 1300 & 1238 & 1083 \\
1 & 3 & 1300 & 1238 & 1083 \\
1 & 6 & 1600 & 1524 & 1334 \\
2 & 1 & 800 & 762 & 667 \\
2 & 2 & 1300 & 1238 & 1083 \\
2 & 3 & 1300 & 1238 & 1083 \\
2 & 6 & 1600 & 1524 & 1334 \\
3 & 0 & 1800 & 1800 & 1800 \\
4 & 0 & 2500 & 2500 & 2500 \\
5 & 0 & 3600 & 3600 & 3600 \\
\hline
\end{tabular}




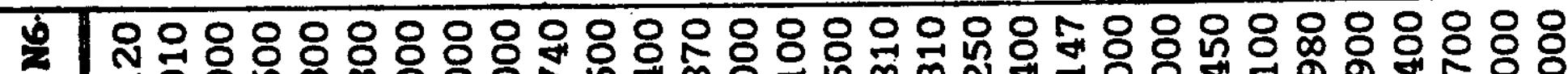

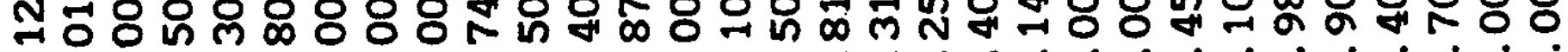
نं $\dot{0}$ भं

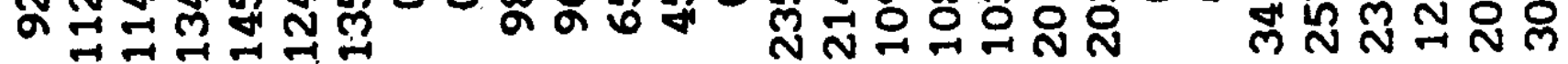

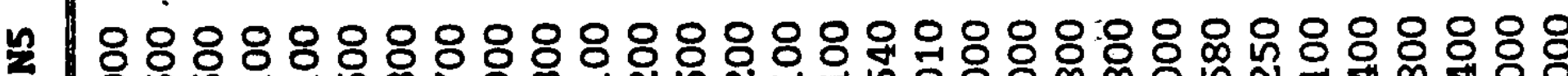

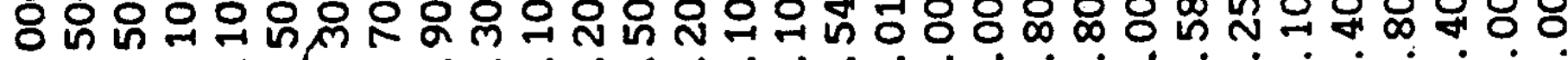

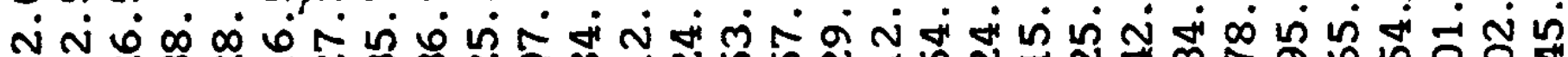

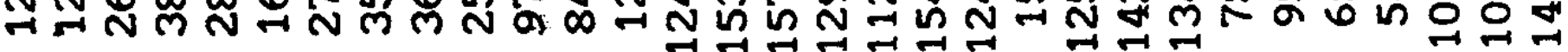

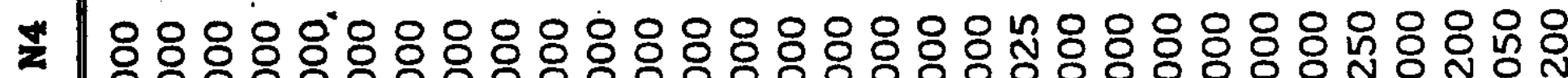

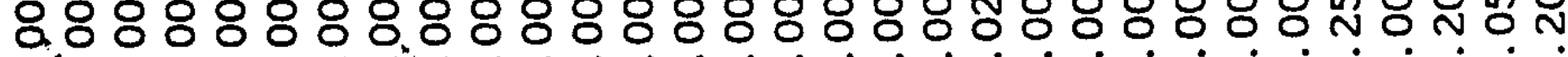

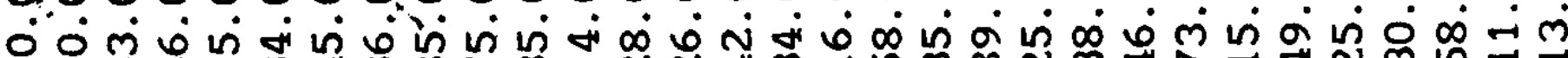

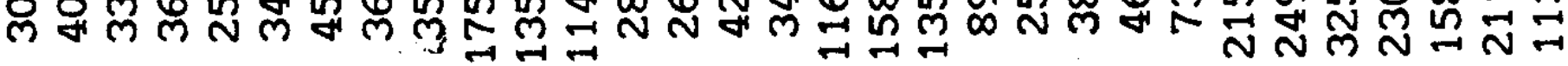

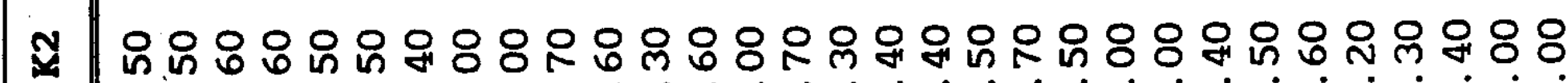

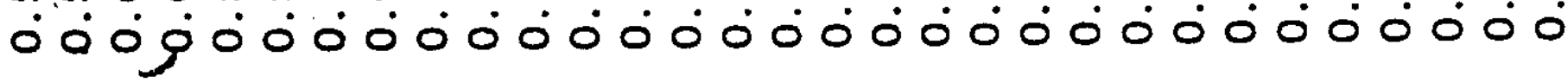

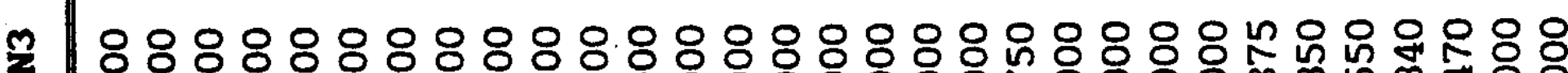
m ن $\dot{0}$ ம

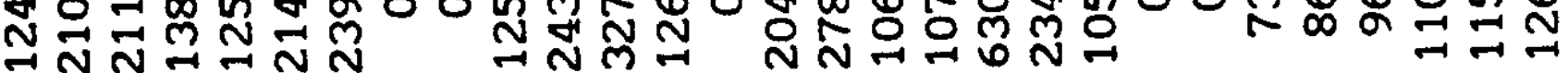

ت

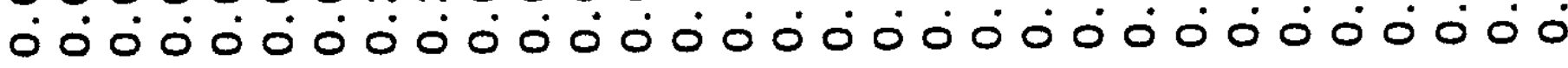

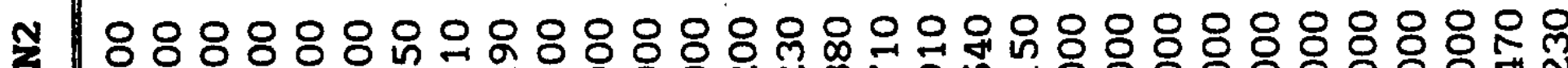

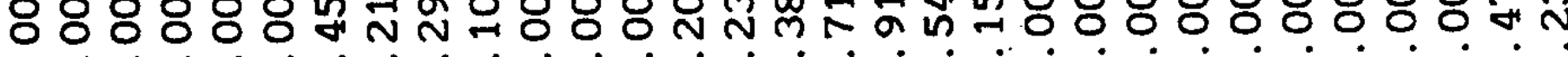

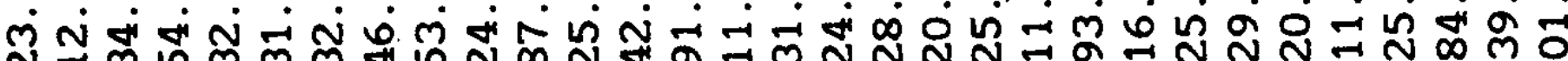

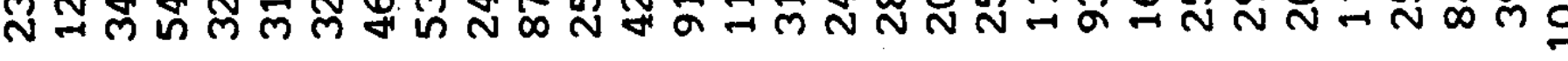

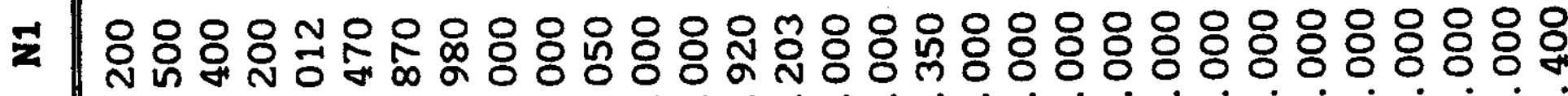

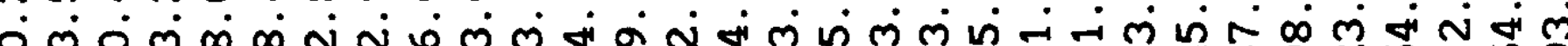

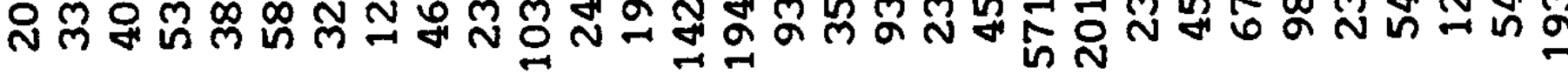

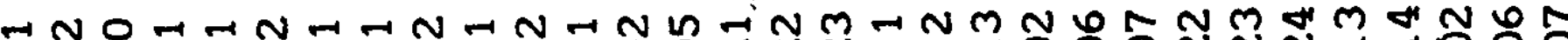

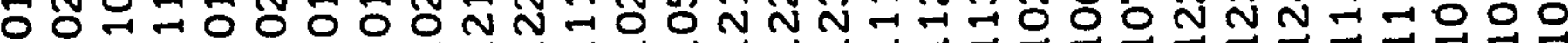
n $\vec{N} \vec{N} \vec{N} \vec{N} \vec{N} \vec{N} \vec{N} \vec{N} \vec{N} \vec{N} \vec{N} \vec{N} \vec{N} \vec{N} \vec{N} \vec{N} \vec{N} \vec{N} \vec{N} \vec{N} \vec{N} \vec{N} \vec{N}$

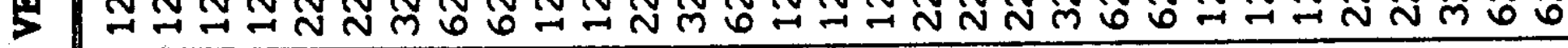

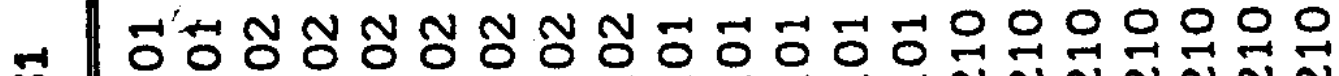
मी $\exists=-7=7=\exists=N N N N N N N N N N N N N N N N N N N N N N$

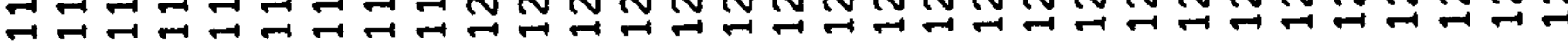


Za svako FMS iz materijalnih formacija oslonjenih jedinica (tabela 3 ), potrebno je izraditi normative vremena po VES-ti, nivou i vidu remonta, kao što je prikazano u tabeli 6 .

Pri tom, kolone $u$ tabeli imaju sledeće značenje:

FMS1 - šifra FMS na koje se normativ odnosi;

VES - šifra VES-ti koja se angažuje na pojedinom nivou i u vidu remonta;

N1 - broj norma-časova godišnje (NC) za TOd FMS u eksploataciji;

N2 - broj NC za TOd FMS u ratnoj rezervi;

K1 - koeficijent angažovanja kvalifikovanih civilnih lica (KV CL) u TOd u miru;

N3 - broj NĆ za TOd u ratu;

K2 - koeficijent realizacije TOd na nivou bataljona (diviziona) u ratu;

N4 - broj NČ za SR FMS u eksploataciji;
N5
- broj NČ za SR FMS ú raf- noj rezervi;
K3 - koeficijent angažovanja KV CL za SR u miru i
N6 - broj NČ za SR FMS u ratu.

\section{Realizacija račnarskog modela}

Posmatranjem funkcionisanja remontnih jedinica LR i SR u miru, pravilnim tumačenjem njihovog ponašanja u različitim uslovima, predviđanjem načina funkcionisanja u ratu, kao i pretpostavljanjem nekih procesa koji nisu prisutni, ali su mogući, dolaz se do teorijskih postavki i koncnpcijskog modela remontne jedinice koji je prikazan na slici 2.

Računarski model remontne jedinice realijovan je na razvijenoj teoriji i u vidu listinga računarskog programa prikazan u prilogu 1. Sastoji se od procedure PRORAS i određenih programskih modula koje procedura PRORAS poziva na sukcesivno izvršavanje.

U odnosu na blok-šeme pojedinih modula programa, prikazane u prvom delu (VTG4/93), program iz priloga $1 \mathrm{je}$,

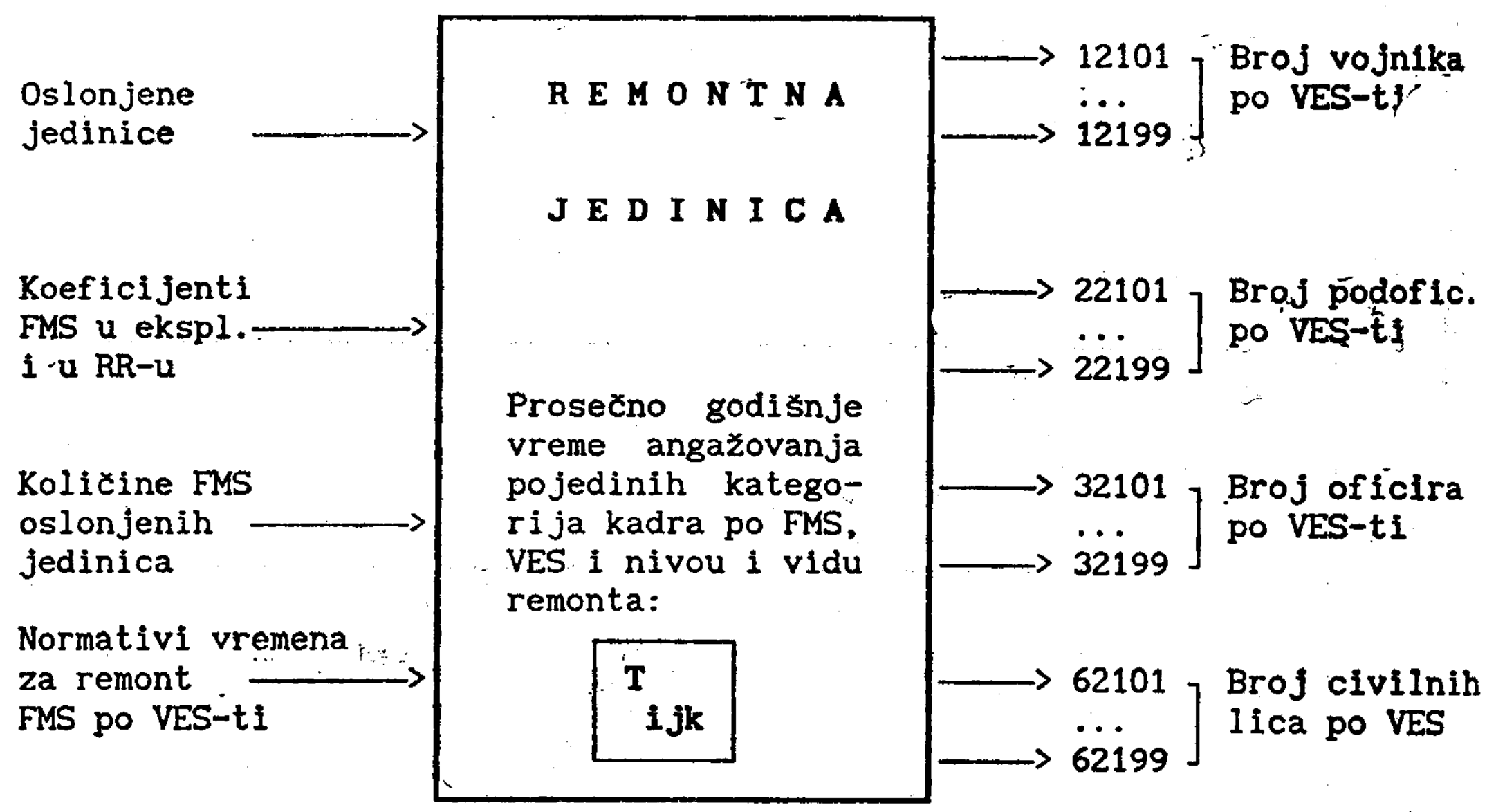

Sl. 2 - Koncepcijski model remontne jedinice 
"u izvesnom smislu, modifikovan i poboljšan. Modul KOEF više ne sadrži instrukcije kojima se zadaju odnosi FMS u eksploataciji i u ratnoj rezervi, već su ti podaci izdvojeni u posebnu tabelu baze podataka (tabela 4), a ona se učitava i pri pozivanju i pri izvršenju samog modula.

Obrasci za proračun potrebnog godišnjeg vrem ena za remont oslonjenih FMS po nivou $i$ vidu remonta i kategorijama kadra

\begin{tabular}{|c|c|c|c|}
\hline NIVO I VID RENONTA & \multicolumn{2}{|c|}{$\underset{\text { KADRA }}{\text { KATEGORIJE }}$} & IZRAZ ZA PRORACUN VREYIDRA \\
\hline \multirow{3}{*}{$\begin{array}{l}1 \text { - Lakl remont } \\
\text { u miru }\end{array}$} & \multicolumn{2}{|c|}{1 - Vojnik } & $S 1=K O L *(N 1 * K E+N 2 * K R R)$ \\
\hline & \multicolumn{2}{|c|}{$\begin{array}{l}2 \text { - Podof } \\
3 \text { - Oficir }\end{array}$} & $S 2=K O L *(N 1 * K E+N 2 * K R R)$ \\
\hline & $6-\mathrm{CL}$ & $\frac{\mathrm{KV}}{\mathrm{VKV}}$ & $\begin{array}{l}\mathrm{S} 1=\mathrm{KOL} * \mathrm{~K} 1 *(\mathrm{~N} 1 * \mathrm{~K} 3+\mathrm{N} 2 * \mathrm{KRR}) \\
\mathrm{S} 2=\mathrm{KOL} *(1-\mathrm{K} 1) *(\mathrm{~N} 4 * \mathrm{KE}+\mathrm{N} 5 * \mathrm{KRR})\end{array}$ \\
\hline \multirow{3}{*}{$\begin{array}{l}\text { 2- Srednji remont } \\
\text { u miru }\end{array}$} & \multicolumn{2}{|c|}{1 - Vojnik } & $S 1=K O L *(N 4 * K E=N 5 * K R R)$ \\
\hline & \multicolumn{2}{|c|}{$\begin{array}{l}2 \text { - Podof } \\
3 \text { - Oficir }\end{array}$} & $S 2=K O L *(N 4 * K E+N 5 * K R R)$ \\
\hline & $6-\mathrm{CL}$ & $\frac{\mathrm{KV}}{\mathrm{V} k \mathrm{v}}$ & $\begin{array}{l}S 1=K O L * K 3 *(N 4 * K E+N 5 * K R R) \\
. S 2=K O L *(1-K 3) *(N 4 * K E+N 5 * K R R)\end{array}$ \\
\hline \multirow{3}{*}{$\begin{array}{l}.3 \text { - Laki remont } \\
\text { u ratu } \\
\text { (nivo b-div) }\end{array}$} & \multicolumn{2}{|c|}{1 - Vojnik } & $\mathrm{S} 1=\mathrm{KOL} * \mathrm{~N} 3 * \mathrm{~K} 2$ \\
\hline & \multicolumn{2}{|c|}{$\begin{array}{l}2=\text { Podof. } \\
3=\text { Oficir }\end{array}$} & $\mathrm{S} 2=\mathrm{KOL} * \mathrm{~N} 3 * \mathrm{~K} 2$ \\
\hline & $6-C$ & $\mathrm{KV}$ & \\
\hline \multirow{3}{*}{$\begin{array}{l}\text { 4- Laki remont } \\
\text { u ratu } \\
\text { (nivo } p-b r \text { ) }\end{array}$} & \multicolumn{2}{|c|}{1 - Vojnik } & Si=KOL*N3 \& S1=KOL*N3*(1-K2) \\
\hline & \multicolumn{2}{|c|}{$\begin{array}{l}2 \text { - Podof. } \\
3 \text { - Oficir }\end{array}$} & $S 2=K O L * N 3$ i S2=KOL*N3*(1-K2) \\
\hline & $6-\mathrm{Cl}$ & $\frac{\mathrm{KV}}{\mathrm{V} K \mathrm{~V}}$ & \\
\hline \multirow{3}{*}{$\begin{array}{l}\text { 5. Srednji remont } \\
\text { u ratu }\end{array}$} & \multicolumn{2}{|c|}{1 - Vojnik } & $\mathrm{S} 1=\mathrm{KOL} * \mathrm{~N} 6 \mathrm{~L}$ \\
\hline & \multicolumn{2}{|c|}{$\begin{array}{l}2 \text { - Podof. } \\
3 \text { - Oficir }\end{array}$} & S2=KOL*N6 \\
\hline & $6-\mathrm{CL}$ & $\frac{K V}{V K V}$ & \\
\hline
\end{tabular}


Način proračuna vremena potrebnog za godišnje održavanje oslonjenih FMS za pojedine kategorije lica, nivoe i vidove remonta prikazan je u tabeli 7 . Modul IZVR je, takođe, izmenjen, tako da se iz tabele 5, pri nejgovom pozivanju i izvršenju, učitavaju podaci o prosečnom godišnjem angažovanju izvršilaca remonta svih kategorija lica za pojedine nivoe i vidove remonta.

Rezultati proračuna, izvršenog sa ulaznim podacima iz tabele $2,3,4,5$ i 6 , prikazani su u prilogu 2, a grupisani su po remontnim jedinicama i kategorijama kadra sa pripadnim suptotalima.

\section{Eksperimentisanje na modelu}

Eksperimentisanje na modelu primenjuje se kada je, zbog složenosti ili ekonomičnosti, ciljeve istraživanja nemoguće ostvariti eksperimentisanjem na realnom sistemu. Remontne jedinice upravo predstavljaju takav slučaj. Naime, za opitovanje realnog sistema bilo bi potrebno utrositi nedopustivo mnogo vremena, što je povezano i sa troškovima, a za remontne jedinice $u$ ratu, to i ne bi bilo moguće u odsutnosti ratnih dejstava.

Prikazani model omogućava izvođenje eksperimenata primenom određenog eksperimentalnog plana. Ako se kao cilj postavi da se ispita uticaj promene trajanja radnog vremena na broj izvršilaca remonta po pojedinim VES-tima, onda eksperimentalni plan podrazumeva da se u zadanim granicama varira srednje vreme angažovanja izvršilaca remonta za sve kategorije lica (tabela 5), a da ostale ulazne vrednosti ostanu nepromenjene.

Prema ovako definisanom eksperimentalnom planu, izvršena su tri eksperimenta $i$ to sa vrednostima srednjeg vremena datog $u$ kolonama VREME, T40 i T35 tabele 5. Eksperimentisano je samo sa jednom remontnom jedinicom SR u miru (43.čSR, šifra »227601«), a upotrebnii rezultati prikazani su $u$ tabeli 8.
Tabela 8

Uporedni rezultati eksperimentisanja za th varijante nedeljnog radnog vremena

\begin{tabular}{|c|c|c|c|c|c|}
\hline \multirow{2}{*}{ VES } & \multirow{2}{*}{$\begin{array}{l}\text { VARI- } \\
\text { JANTA }\end{array}$} & \multicolumn{2}{|c|}{ BROJ IZVRSILACA } & \multicolumn{2}{|c|}{ OSTATAK ZAOKR: } \\
\hline & & $\mathbf{K V}$ & VKV & OST1 & $\operatorname{osi2} 2$ \\
\hline 12101 & $\begin{array}{l}\text { v1 } \\
\text { v2 } \\
\text { v3 }\end{array}$ & $\begin{array}{l}65 \\
67 \\
78\end{array}$ & $\begin{array}{l}0 \\
0 \\
0\end{array}$ & $\begin{array}{l}0.79 \\
0.30 \\
0.90\end{array}$ & $\begin{array}{l}0.00 \\
0.00 \\
0.00\end{array}$ \\
\hline 12102 & $\begin{array}{l}\text { v1 } \\
\text { v2 } \\
\text { v3 }\end{array}$ & $\begin{array}{l}81 \\
83 \\
97\end{array}$ & $\begin{array}{l}0 \\
0 \\
0\end{array}$ & $\begin{array}{l}0.24 \\
0.11 \\
0.44\end{array}$ & $\begin{array}{l}0.00 \\
0.00 \\
0.00\end{array}$ \\
\hline 12110 & $\begin{array}{l}v_{1} \\
v_{2} \\
v_{3}\end{array}$ & $\begin{array}{l}57 \\
59 \\
69\end{array}$ & $\begin{array}{r}0 \\
0 \\
0 \\
0\end{array}$ & $\begin{array}{l}0.78 \\
0.11 \\
0.30\end{array}$ & $\begin{array}{l}0.00 \\
0.00 \\
0.00\end{array}$ \\
\hline 12111 & $\begin{array}{l}71 \\
12 \\
V 3\end{array}$ & $\begin{array}{l}70 \\
72 \\
84\end{array}$ & $\begin{array}{l}0 \\
0 \\
0\end{array}$ & $\begin{array}{l}0.40 \\
0.02 \\
0.44\end{array}$ & $\begin{array}{l}0.00 \\
0.00 \\
0.00\end{array}$ \\
\hline 12121 & $\begin{array}{l}11 \\
12 \\
13\end{array}$ & $\begin{array}{l}40 \\
41 \\
48\end{array}$ & $\begin{array}{l}0 \\
0 \\
0\end{array}$ & $\begin{array}{l}0.54 \\
0.48 \\
0.63\end{array}$ & $\begin{array}{l}0.00 \\
0.00 \\
0.00\end{array}$ \\
\hline 12122 & $\begin{array}{l}v_{1} \\
\sqrt{2} \\
\text { v3 }\end{array}$ & $\begin{array}{l}46 \\
47 \\
55\end{array}$ & $\begin{array}{l}0 . \\
0 \\
0\end{array}$ & $\begin{array}{l}0.21 \\
0.28 \\
0.43\end{array}$ & $\begin{array}{l}0.00 \\
0.00 \\
0.00\end{array}$ \\
\hline KAT: 1 & $\begin{array}{l}\text { v1 } \\
\text { v2 } \\
\text { v3 }\end{array}$ & $\begin{array}{l}359 \\
369 \\
431\end{array}$ & $\begin{array}{l}0 \\
0 \\
0\end{array}$ & $\begin{array}{l}2.96 \\
1.30 \\
3.14\end{array}$ & $\begin{array}{l}0.00 \\
0.00 \\
0.00\end{array}$ \\
\hline 22101 & $\begin{array}{l}\text { V1 } \\
\text { v2 } \\
\text { v3 }\end{array}$ & $\begin{array}{l}0 \\
0 \\
0\end{array}$ & $\begin{array}{l}30 \\
32 \\
37\end{array}$ & $\begin{array}{l}0.00 \\
0.00 \\
0.00\end{array}$ & $\begin{array}{l}0.89 \\
0.44 \\
0.09\end{array}$ \\
\hline 22102 & $\begin{array}{l}v_{1} \\
\text { V2 } \\
\text { v3 }\end{array}$ & $\begin{array}{l}0 \\
0 \\
0\end{array}$ & $\begin{array}{l}31 \\
32 \\
37\end{array}$ & $\begin{array}{l}0.00 \\
0.00 \\
0.00\end{array}$ & $\begin{array}{l}0.30 \\
0.87 \\
0.58\end{array}$ \\
\hline 22111 & $\begin{array}{l}\text { v1 } \\
\text { v2 } \\
\text { v3 }\end{array}$ & $\begin{array}{l}0 \\
0 \\
0\end{array}$ & $\begin{array}{l}24 \\
25 \\
29\end{array}$ & $\begin{array}{l}0.00 \\
0.00 \\
0.00\end{array}$ & $\begin{array}{l}0.28 \\
0.49 \\
0.14\end{array}$ \\
\hline KAT: 2 & $\begin{array}{l}\text { v1 } \\
\text { v2 } \\
\text { v3 }\end{array}$ & $\begin{array}{l}0 \\
0 \\
0\end{array}$ & $\begin{array}{r}85 \\
89 \\
103\end{array}$ & $\begin{array}{l}0.00 \\
0.00 \\
0.00\end{array}$ & $\begin{array}{l}1.47 \\
1.80 \\
0.81\end{array}$ \\
\hline 32101 & $\begin{array}{l}\text { V1 } \\
\text { V2 } \\
\text { V3 }\end{array}$ & $\begin{array}{l}0 \\
0 \\
0\end{array}$ & $\begin{array}{l}44 \\
46 \\
52\end{array}$ & $\begin{array}{l}0.00 \\
0.00 \\
0.00\end{array}$ & $\begin{array}{l}0.13 \\
0.34 \\
0.97\end{array}$ \\
\hline 32102 & $\begin{array}{l}\text { V1 } \\
\text { v2 } \\
\text { v3 }\end{array}$ & $\begin{array}{l}0 \\
0 \\
0\end{array}$ & $\begin{array}{l}4 \\
5 \\
5\end{array}$ & $\begin{array}{l}0.00 \\
0.00 \\
0.00\end{array}$ & $\begin{array}{l}0.99 \\
0.24 \\
0.99\end{array}$ \\
\hline KAT: 3 & $\begin{array}{l}\text { v1 } \\
\text { v2 } \\
\text { v3 }\end{array}$ & $\begin{array}{l}0 \\
0 \\
0 \\
\end{array}$ & $\begin{array}{l}48 \\
51 \\
57 \\
\end{array}$ & $\begin{array}{l}0.00 \\
0.00 \\
0.00\end{array}$ & $\begin{array}{l}1.12 \\
0.58 \\
1.96\end{array}$ \\
\hline 62101 & $\begin{array}{l}\text { v1 } \\
\text { v2 } \\
\text { v3 }\end{array}$ & $\begin{array}{l}13 \\
14 \\
16\end{array}$ & $\begin{array}{l}20 \\
21 \\
24\end{array}$ & $\begin{array}{l}0.69 \\
0.38 \\
0.43\end{array}$ & $\begin{array}{l}0.54 \\
0.57 \\
0.64\end{array}$ \\
\hline 62102 & $\begin{array}{l}\text { v1 } \\
\text { v2 } \\
\text { v3 }\end{array}$ & $\begin{array}{l}17 \\
17 \\
20\end{array}$ & $\begin{array}{l}17 \\
17 \\
20\end{array}$ & $\begin{array}{l}0.08 \\
0.93 \\
0.49\end{array}$ & $\begin{array}{l}0.08 \\
0.93 \\
0.49\end{array}$ \\
\hline 62105 & $\begin{array}{l}\text { V1 } \\
\text { V2 } \\
\text { V3 }\end{array}$ & $\begin{array}{r}8 \\
9 \\
10\end{array}$ & $\begin{array}{l}5 \\
6 \\
7\end{array}$ & $\begin{array}{l}0.76 \\
0.20 \\
0.51\end{array}$ & $\begin{array}{l}0.84 \\
0.13 \\
0.01\end{array}$ \\
\hline KAT: 6 & $\begin{array}{l}\text { v1 } \\
\text { v2 } \\
\text { v3 }\end{array}$ & $\begin{array}{l}38 \\
40 \\
46 \\
\end{array}$ & $\begin{array}{l}42 \\
44 \\
51 \\
\end{array}$ & $\begin{array}{l}1.53 \\
1.51 \\
1.43\end{array}$ & $\begin{array}{l}1.46 \\
1.63 \\
1.14\end{array}$ \\
\hline UKUPNO & $\begin{array}{l}\text { v1 } \\
\text { v2 } \\
\text { v3 }\end{array}$ & $\begin{array}{l}397 \\
409 \\
477\end{array}$ & $\begin{array}{l}175 \\
184 \\
211\end{array}$ & $\begin{array}{l}4.49 \\
2.81 \\
4.57\end{array}$ & $\begin{array}{l}4.05 \\
4.01 \\
3.91\end{array}$ \\
\hline
\end{tabular}


'Pri tom su V1, V2 i V3 varijante iz plana eksperimenata za nedeljno radno vreme $\mathrm{T}=42, \mathrm{~T}=40$ i $\mathrm{T}=35$ časova, respektivno.

Na primeru VES-ti »12122« može se uočiti da skraćenje nedeljnog radnog vremena za 2 sata zahteva uvođenje još jednog izvršioca, a skraćenje za 7 sati čak 9 dodatnih izvršilaca. Boljom organizacijom radnog dana može se, bez većih zahvata, kompenzovati radno vreme jednog izvršioca, ali za 9 izvršioca to nije moguce. Slične analize mogu se sprovesti i za ostale VES-ti. Dakle, može se zaključiti da pri prelasku sa 42-časovnog nedeljnog radrog vremena na 40-Casovno nije potrebno menjati ličnu formaciju posmatrane remontne jedinice, ali je potrebno povesti više računa o boljem iskorišćenju radnog vremena.

Koristeći prikazanu metodologiju eksperimentisanja, mogu se formirati različiti eksperimentalni planovi, u zavisnosti od postavljjenog cilja istraživanja. Variranje vrednosti ulaznih faktora obično se vrši na dva, a najviše na

\section{Literatura:}

[1] N. Bračika: sIzrada normativa proizvodne radne snagex; VTG 4/86, Beograd, 1986.

[2] sProgramski sistem za proračun proizvodne radne snage jedinica 1 ustanova TSL KoV JNA u miru 1 ratuk;projektni zadatak, CVTS KoV JNA General armije Ivan Gošnjakı, Zagreb, 1985.

[3] I. Bračika: sMetodoloski pristup određivanju radne snage tehnicke radionice primenom ra- tri nivoa. Mogu se koristiti kombinacije nekoliko faktora, a drugi se drže nepromenjeni.

\section{Zaključak}

U članku je dat prikaz programskog sistema za proračun projzvodne radne snage remontnih jedinica realizovan u dBASE IV, a formiranje baze ulaznih podataka i proračun izvršeni su na hipotetičkom primeru. Posmatrajući program kao računarski model remontne jedinice, prikazan je način eksperimentisanja njime, a radi provere adekvatnosti lične formacije posmatrane jedinice pri skraćenju radne nedelje sa 42 na 40 radnih časova. Rezultati eksperimenata pokazuju da postojeća lična formacija zadovoljava. Pošto se radi o linearnim zavisnostima ulaznih i izlaznih veličina moddela, induktivnim zaključivanjem se rezultati mogu uopštiti i primeniti na sve ostale remontne jedinice koje funkcionišu $u$ istim zadatim uslovima.

Cunarski zasnovanog modelovanjar; magistarski rad, ETF Sarajevo, 1985.

[4] N. Bračika: sProgramski sistem za proracun proizvodne radne snage remontnih jedinica I ustanova VJא; VTG 4/93, Beograd, 1993.

[5] V. Žiljak, G. Smiljanić: sodeliranje 1 simuliranje sa racunarimax; ETF Zagreb, 1980. 


\section{Page \# 1}

* Program : PRORAS.PRG - program za proracun

* $\quad$ proizvodne radne snage

* Autor : ppk mr N. BRACIKA, dipl. ing.

* Poziva ga: PRS MENI

* On poziva: KOE $\bar{F}$, SUMKOE, VRREM, SUMAVR i IZVR

PROCEDURE PRORAS

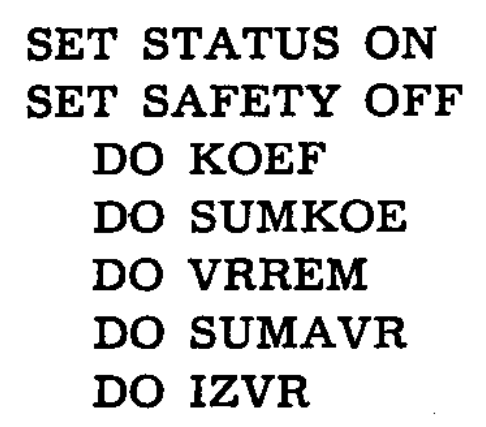

RETURN TO MASTER

* Modul : KOEF - određivanje koeficijenata

* eksploatacije za FMS

* Poziva ga : PRORAS.PRG

* Ulazne baze : DATOSL, DATFMS I KOEF

* Izlazne baze: POMOCNA

PROCEDURE KOEF

DECLARE A [8], B [3], C [6], D [16,4]

STORE " "TO FMS M, A [1], A [2], A [3], A [4], A [5], A [6], A [7], A [8]

STORE " "TO B [1], B [2], C[1], C [2], C [3]

STORE 0 TO KE, KRR, B [3], C [4], C [5], C [6]

SELECT 9

USE KOEF

$N=$ RECCOUNT ( )

COPY TO ARRAY D NEXT N

SELECT 3

USE POMOCNA

ZAP

USE DATFMS IN 2

SELECT 1

USE DATOSL

COPY TO ARRAY A NEX' 1

DO WHILE .T.

SELECT 2

SET FILTER TO SIF $=A$ [7]

GO TOP

COPY TO ARRAY B NEXT 1

DO WHILE .NOT. EOF ()

FMS $M=\operatorname{SUBSTR}(B[2], 1,3)$ 
IF FMS M $M<>121 "$.AND.FMS_M $<>$ "122".AND.FMS_ $M<>$ "123"

ENDIF FMS $m=" 999 "$

$\mathrm{KLJUC}=\mathrm{FMS} \mathbf{M}+\mathbf{A}[8]$

$\mathrm{I}=\mathbf{0}$

DO WHILE $\mathrm{I}<\mathrm{N}$

$\mathrm{I}=\mathrm{I}+1$

IF $\mathrm{KLJUC}=\mathrm{D}[\mathrm{I}]+,\mathrm{D}[\mathrm{I}, 2]$

$C[5]=D[I, 3]$

$C[6]=D[I, 4]$

ENDIF

ENDDO

$C$ [3] $=B$ [2]

$C[4]=B$ [3]

SELECT 3

DO CASE

CASE A [5] $<>$ "

".AND.A [5] ><"00000000"

$\mathbf{C}[1]=\mathbf{A}[5]$

$\mathrm{C}[2]=\mathrm{A}[6]$

APPEND FROM ARRAY C

$\mathrm{C}[1]=\mathrm{A}$ [3]

$C[2]=A[4]$

APPEND FROM ARRAY C

$\mathrm{C}[1]=\mathrm{A}[1]$

$C[2]=A$ [2]

APPEND FROM ARRAY C

CASE $A[3]<>" \quad$ ".AND.A [3] $><" 00000000 "$

$\mathrm{C}[1]=\mathrm{A}[3]$

$C[2]=A[4]$

APPEND FROM ARRAY C

$\mathrm{C}[1]=\mathrm{A}[1]$

$C[2]=A[2]$

APPEND FROM ARRAY C

OTHERWISE

$\mathrm{C}[1]=\mathrm{A}[1]$

$C[2]=A[2]$

APPEND FROM ARRAY C

ENDCASE

SELECT 2

SKIP

IF .NOT. EOF ()

COPPY TO ARRAY B NEXT 1

LOOP

ENDIF

EXIT

ENDDO

SET FILTER TO

SELECT 1

SKIP

IF .NOT. EOF () 


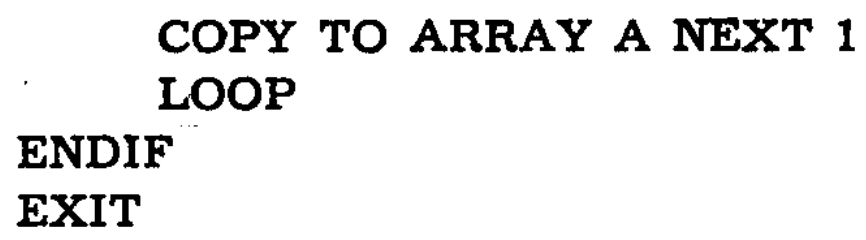

ENDDO

CLOSE DATA

\section{RETURN}

- Modul : SUMKOE - nalaženje srednjih koeficijenata FMS u eksploataciji 1 u RR-u

- Poziva ga : PRORAS. PRg

Page \# 3

* On poziva : SUMKOE.QBE

* Ulazne baze : POMOCNA

* Izlazne baze: DFMSOSL

PROCEDURE SUMKOE

SET VIEV TO QBE/SUMKOE

COPY TO DFMSOSL

CLOSE DATA

\section{RETURN}

* Modul : VRREM - proracun potrebnog vremena za odrzavanje oslonjenih FMS

* Poziva ga : PRORAS.PRG

* Ulazne baze : DATOSL, DATNORM, DFMSOSL

* Izlazne baze: DATVRP

\section{PROCEDURE VRREM}

DECLARE D [6], E [11], F [5], A [8]

STORE " " TO A [1], A [2], A [3], A [4], A [5], A [6], A [7], A [8]

STORE " " TO D [1], D [2], D [3], E [1], E [2], F [1], F [2], F [3], KAT

STORE 0 TO D [4], D [5], D [6], F [4], F [5], S1M, S2M

SELECT 6

USE DATVRP

ZAP

USE DATNORM IN 3

USE DATOSL IN 1 ORDER DX 1

SELECT 5

USE DFMSOSL

DO WHILE .NOT. EOF()

COPY TO ARRAY D NEXT 1

SELECT 3

SET FILTER TO FMS1 $=\mathrm{D}$ [3]

GO TOP

DO WHILE .NOT. EOF ()

COPY TO ARRAY E NEXT 1

$\mathrm{KAT}=\operatorname{SUBSTR}(\mathrm{E}[2], 1,1)$ 
DO CASE

CASE D [2] ="1"

DO CASE

CASE KAT $=" 1 "$

$$
\begin{aligned}
& S 1 M=D[4] *(E[3] * D[5]+E[4] * D[6]) \\
& S 2 M=0
\end{aligned}
$$

CASE KAT $=$ "2".OR.KAT $=$ "3"

$\mathrm{S} 1 \mathrm{M}=0$

$S 2 M=D[4] *(E[3] * D[5]+E[4] * D[6])$

CASE KAT $=" 6 "$

$$
\begin{aligned}
& S 1 M=D[4] * E[5] *(E[3] * D[5]+E[4] * D[6]) \\
& S 2 M=D[4] *(1-E[5]) *(E[3] * D[5]+E[4] * D[6])
\end{aligned}
$$

\section{ENDCASE}

CASE D [2]="2"

DO CASE

CASE KAT $=" 1 "$

$$
\begin{aligned}
& \mathrm{S} 1 \mathrm{M}=\mathrm{D}[4] *(\mathrm{E}[8] * \mathrm{D}[5]+\mathrm{E}[9] * \mathrm{D}[6]) \\
& \mathrm{S} 2 \mathrm{M}=0
\end{aligned}
$$

CASE KAT $=$ "2".OR.KAT $=$ "3"

$\mathrm{S} 1 \mathrm{M}=\mathbf{0}$

$$
S 2 M=D[4] *(E[8] * D[5]+E[9] * D[6])
$$

CASE KAT $=$ "6"

$$
S 1 M=D[4] * E[10] *(E[8] * D[5]+E[9] * D[6])
$$

ENDCASE

$$
\mathrm{S} 2 \mathrm{M}=\mathrm{D}[4] *(1-\mathrm{E}[10]) *(\mathrm{E}[8] * \mathrm{D}[5]+\mathrm{E}[9] * \mathrm{D}[6])
$$

CASE D [2] ="3"

Page \# 4

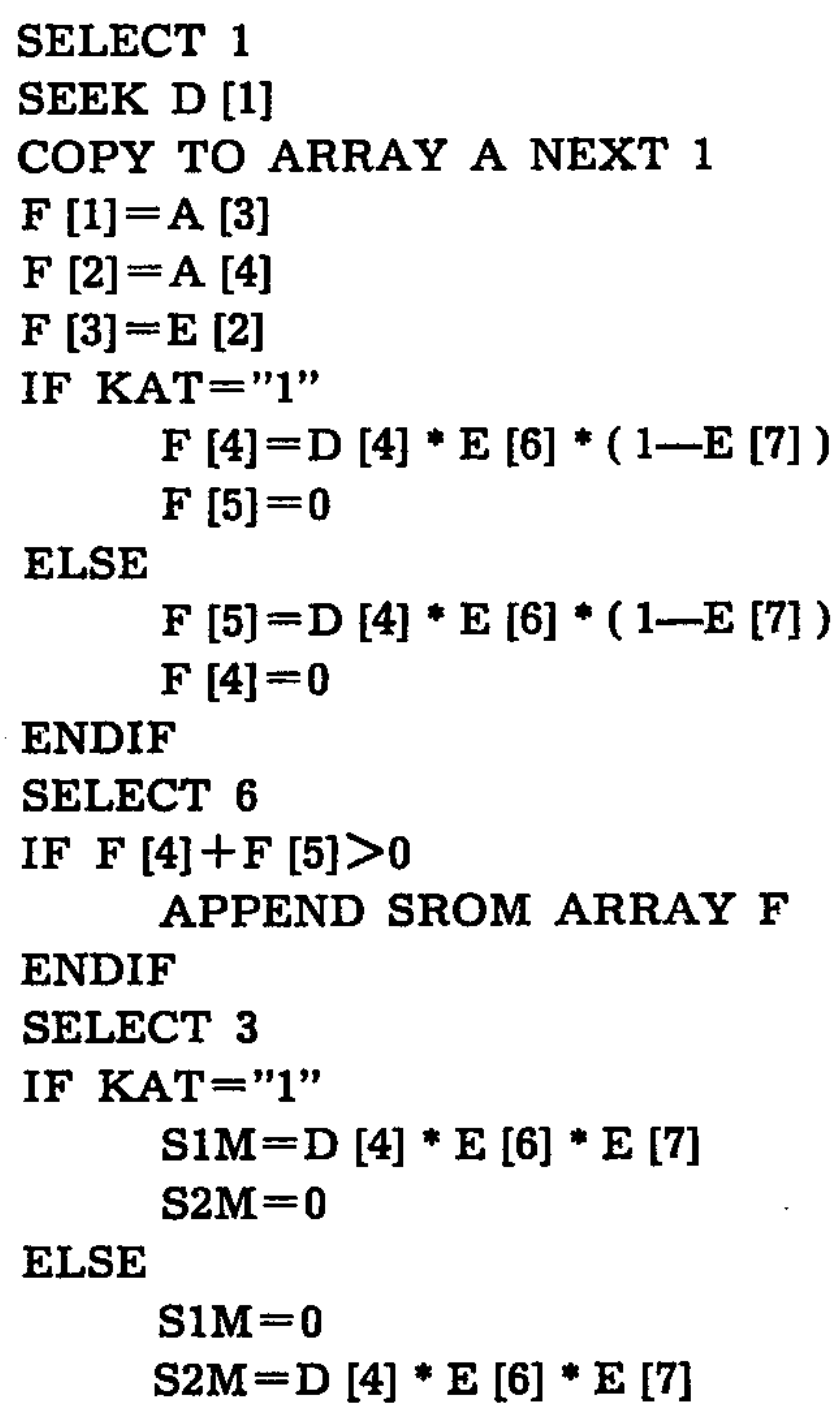


ENDIF

CASE D [2] = "4"

IF KAT $=" 1 "$

$$
\begin{aligned}
& S 1 M=D[4] * E[6] \\
& S 2 M=0
\end{aligned}
$$

ELSE

$$
\begin{aligned}
& \mathrm{S} 1 \mathrm{M}=0 \\
& \mathrm{~S} 21 \mathrm{M}=\mathrm{D}[4] * \mathrm{E}[6]
\end{aligned}
$$

ENDIF

CASE D [2] = "5"

IF $\mathrm{KAT}=" 1$ "

$$
\begin{aligned}
& \mathrm{S} 1 \mathrm{M}=\mathrm{D}[4] * \mathrm{E}[11] \\
& \mathrm{S} 2 \mathrm{M}=0
\end{aligned}
$$

ELSE

$$
\begin{aligned}
& \mathrm{S} 1 \mathrm{M}=0 \\
& \mathrm{~S} 2 \mathrm{M}=\mathrm{D}[4] * \mathrm{E}[11]
\end{aligned}
$$

ENDIF

$$
\begin{aligned}
& \text { ENDCASE } \\
& F[1]=D[1] \\
& F[2]=D[2] \\
& F[3]=E[2] \\
& F[4]=S 1 M \\
& F[5]=S 2 M \\
& \text { SELECT } 6 \\
& \text { IF } F[4]+F[5]>0
\end{aligned}
$$

APPEND FROM ARRAY F

ENDIF

STORE " " TO F [1], F [2], F [3]

STORE 0 TO F [4], F [5]

SELECT 3

SKIP 1

ENDDO

SET FILTER TO

SELECT 5

SKIP 1

ENDDO

CLOSE DATA

\section{RETURN}

\section{Page \# 5}

* Modul : SUMAVR - sumiranje vremena po rem. jedinicama,

\section{* nivou i vidu remonta i VES-ti}

- Poziva ga : PRORAS.PRG

- On poziva : SUMAVR.QBE

* Ulazne baze : DATVRP

* Izlazne baze: DATVR

PROCEDURE SUMAVR

SET VIEW TO QBE/SUMAVR

COPY TO DAVR.DBF

CLOSE DATA

\section{RETURN}


- Modul : IZVR - proracun izvrsilaca remonta

- Poziva ga : PRORAS.PRG

- Ulazne baze : DATVR i VREME

- Izlazne baze: DATIZV

PROCEDURE IZVR

DECLARE D [11,3], G [5], H [7]

STORE " "TO G [1], G [2], G [3], H [1], H [2], H [3], KAT

STORE 0 TO G [4], G [5], H [4], H [5], H [6], H [7], T, B1, B2, OST1, OST2

SELECT 9

USE VREME

$\mathrm{N}=$ RECCOUNT ( )

COPY TO ARRAY D NEXT $N$

SELECT 8

USE DATIZV

ZAP

SELECT 7

USE DATVR

DO WHILE .NOT. EOF ()

COPY TO ARRAY G NEXT 1

KAT $=$ SUBSTR ( G [2] >"2"

ENDIF

$\mathrm{KAT}=$ "0"

$\mathrm{KLJUC}=\mathrm{G}[2]+\mathrm{KAT}$

$\mathrm{I}=0$

DO WHILE I $<N$

$\mathrm{I}=\mathrm{I}+1$

IF $\mathrm{KLJUC}=\mathrm{D}[\mathrm{I}, 1]+\mathrm{D}[\mathrm{I}, 2]$

$\mathrm{T}=\mathrm{D}[\mathrm{I}, 3]$

EXIT

ENDIF

ENDDDO

$\mathrm{H}[1]=\mathrm{G}[1]$

$H[2]=G[2]$

$\mathrm{H}[\mathbf{3}]=\mathrm{G} \cdot[3]$

$\mathrm{H}[4]=\mathrm{INT}(\mathrm{G}[4] / \mathrm{T})$

$H[5]=I N T(G[5] / T)$

$\mathrm{H}[6]=\mathrm{G}[4] / \mathrm{T}-\mathrm{H}[4]$

$\mathrm{H}[7]=\mathrm{G}[5] / \mathrm{T}-\mathrm{H}[5]$

SELECT 8

APPEND FROM ARRAY H

SELECT 7

SKIP 1

ENDDO

CLOSE DATA

RETURN

- K_ Kraj programa PRORAs. PRG 
Rezultati proračuna broja izvršilaca za $T=42$ sata

PRORACUNATI BROJ IZVRSILACA Str: 1

\begin{tabular}{|c|c|c|c|c|}
\hline \multirow{2}{*}{ VE S } & \multicolumn{2}{|c|}{ Broj izvršilaca } & \multicolumn{2}{c|}{ Ostak zaokruz } \\
\cline { 2 - 5 } & $\mathrm{KV}$ & $\mathrm{VKV}$ & $\mathrm{KV}$ & $\mathrm{VKV}$ \\
\hline
\end{tabular}

13. VTO

\begin{tabular}{lrrrr}
\hline 12101 & 37 & 0 & 0.72 & 0.00 \\
12102 & 35 & 0 & 0.51 & 0.00 \\
12110 & 44 & 0 & 0.60 & 0.00 \\
12111 & 65 & 0 & 0.49 & 0.00 \\
12121 & 1 & 0 & 0.91 & 0.00 \\
12122 & 7 & 0 & 0.56 & 0.00 \\
Svega: & 189 & 0 & 3.79 & 0.00 \\
22101 & 0 & 25 & 0.00 & 0.83 \\
22102 & 0 & 31 & 0.00 & 0.52 \\
22111 & 0 & 1 & 0.00 & 0.22 \\
Svega: & 0 & 57 & 0.00 & 1.57 \\
32101 & 0 & 24 & 0.00 & 0.49 \\
32102 & 0 & 1 & 0.00 & 0.62 \\
Svega: & 0 & 25 & 0.00 & 1.11 \\
62101 & 5 & 15 & 0.02 & 0.06 \\
62102 & 7 & 23 & 0.68 & 0.05 \\
62105 & 2 & 2 & 0.27 & 0.27 \\
Svega: & 14 & 40 & 0.97 & 0.38 \\
SVEGA: & 203 & 122 & 4.76 & 3.06
\end{tabular}

92. VTO

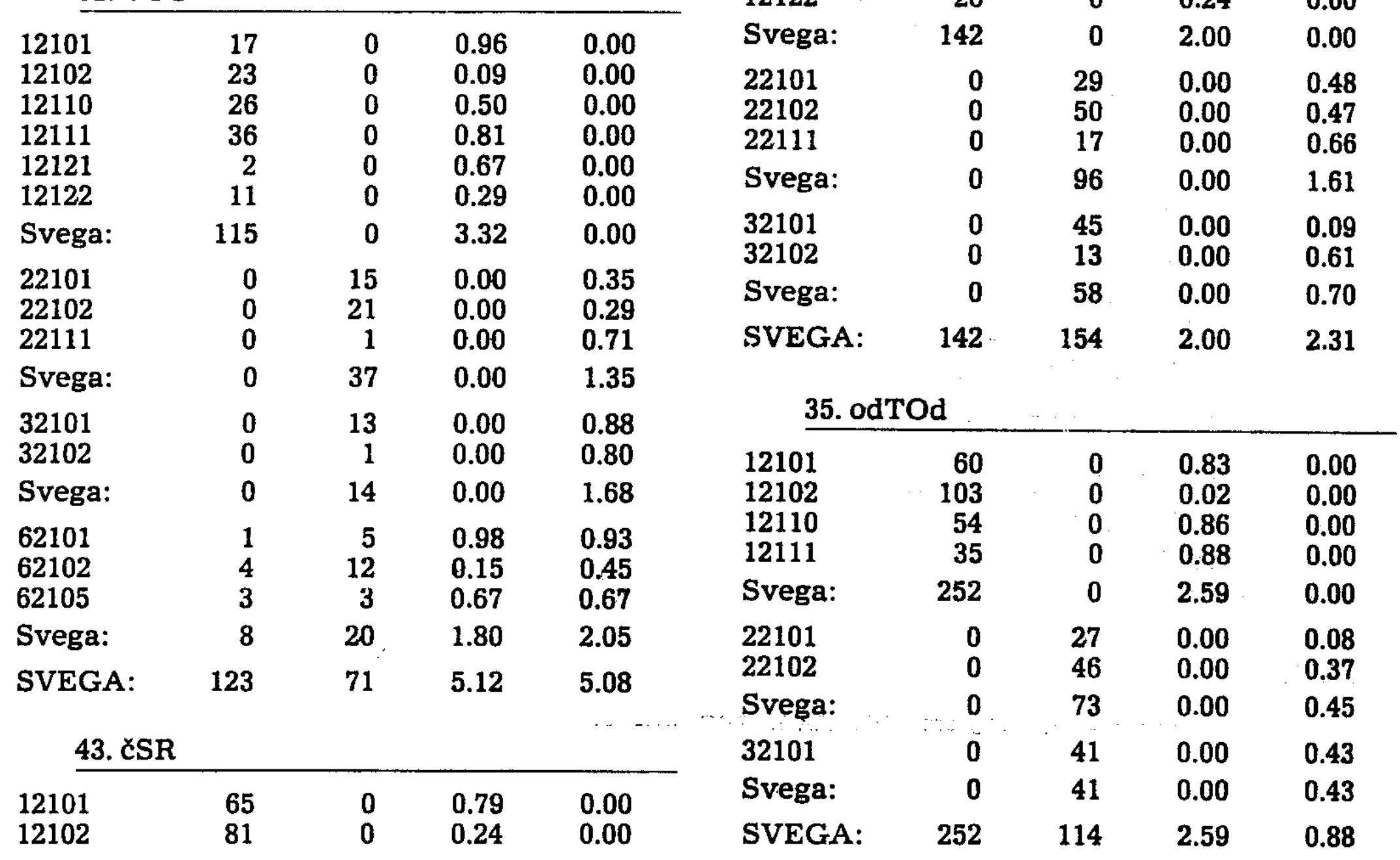




\begin{tabular}{|c|c|c|c|c|}
\hline \multirow{2}{*}{ VE S } & \multicolumn{2}{|c|}{ Broj } & izvrsilaca & \multicolumn{2}{c|}{ Ostak zaokruz } \\
\cline { 2 - 5 } & KV & VKV & KV & VKV \\
\hline
\end{tabular}

52. VTO

\begin{tabular}{lrrrr}
\hline 12101 & 133 & 0 & 0.99 & 0.00 \\
12102 & 226 & 0 & 0.93 & 0.00 \\
12110 & 192 & 0 & 0.48 & 0.00 \\
12111 & 125 & 0 & 0.89 & 0.00 \\
12121 & 21 & 0 & 0.06 & 0.00 \\
12122 & 44 & 0 & 0.09 & 0.00 \\
Svega: & 741 & 0 & 3.44 & 0.00 \\
22101 & 0 & 40 & 0.00 & 0.73 \\
22102 & 0 & 69 & 0.00 & 0.72 \\
22111 & 0 & 29 & 0.00 & 0.67 \\
Svega: & 0 & 138 & 0.00 & 2.12 \\
32101 & 0 & 93 & 0.00 & 0.44 \\
32102 & 0 & 6 & 0.00 & 0.53 \\
Svega: & 0 & 99 & 0.00 & 0.97 \\
SVEGA: & 741 & 237 & 3.44 & 3.09
\end{tabular}

\begin{tabular}{|c|c|c|c|c|}
\hline \multirow{2}{*}{ VES } & \multicolumn{2}{|c|}{ Broj izvršilaca } & \multicolumn{2}{|c|}{ Ostak zaokruz } \\
\hline & KV & VKV & KV & VKV \\
\hline
\end{tabular}

72. CSR

\begin{tabular}{lrrrr}
\hline 12101 & 46 & 0 & 0.09 & 0.00 \\
12102 & 56 & 0 & 0.04 & 0.00 \\
12110 & 51 & 0 & 0.59 & 0.00 \\
12111 & 60 & 0 & 0.86 & 0.00 \\
12121 & 8 & 0 & 0.89 & 0.00 \\
12122 & 8 & 0 & 0.69 & 0.00 \\
Svega: & 229 & 0 & 3.16 & 0.00 \\
22101 & 0 & 65 & 0.00 & 0.75 \\
22102 & 0 & 56 & 0.00 & 0.47 \\
22111 & 0 & 5 & 0.00 & 0.89 \\
Svega: & 0 & 126 & 0.00 & 2.11 \\
32101 & 0 & 61 & 0.00 & 0.09 \\
32102 & 0 & 4 & 0.00 & 0.13 \\
Svega: & 0 & 65 & 0.00 & 0.22 \\
SVEGA: & 229 & 191 & 3.16 & 2.33
\end{tabular}

